\title{
Sampled-Data Backstepping Control of a Quadrotor Unmanned Aerial Vehicle
}

\author{
Abdul Jabbar, Fahad Mumtaz Malik \\ Department of Electrical Engineering, College of Electrical and Mechanical Engineering, \\ National University of Sciences and Technology, Islamabad, Pakistan.
}

\begin{abstract}
Article Info
Article history:

Received Feb 9, 2015

Revised Apr 25, 2015

Accepted May 12, 2015

\section{Keyword:}

Backstepping control Euler Approximate Model

ABSTRACT

Sampled-data backstepping control of a quadrotor UAV is presented in this paper. The discrete time controllers have been designed on the basis of a continuous time system model and an approximate discrete time equivalent system model (Euler Approximate model) obtained using the Euler method. The performance of the controllers obtained using the two sampled-data approaches has been compared in the presence of wing gusts and modeling uncertainties. Simulations have revealed that the backstepping controller designed on the basis of an approximate discrete time model has better performance in the presence of wind gusts and modeling uncertainties. In addition, the closed loop system has a larger region of attraction.
\end{abstract}

Region of Attraction

Sampled-Data control

UAV

\section{Copyright $@ 2015$ Institute of Advanced Engineering and Science.}

All rights reserved.

\section{Corresponding Author:}

Abdul Jabbar,

Department of Electrical Engineering,

College of Electrical and Mechanical Engineering,

National University of Sciences and Technology, Islamabad, Pakistan.

Email: abduljabbar71@ee.ceme.edu.pk

\section{INTRODUCTION}

The stability, robustness and cost effectiveness of digital electronics has made applications of microcontrollers an integral part of every modern control system [1] [2]. The digital control of the dynamic systems, termed as sampled-data control has been one of the focal points in control systems research in the recent past.

The control systems literature generally discusses two frameworks for the design of digital controllers for a continuous time dynamic system. The first approach suggests the design of a continuous time controller for a continuous time system which is subsequently discretized. In the second approach, the continuous time system model is discretized and a discrete time controller is designed on the basis of an approximate discrete time equivalent system model [1] [3].

In case of linear systems, the discrete time equivalent model is conveniently obtained by integrating a continuous time model over the sampling period. However in case of nonlinear systems, the discrete time equivalent model cannot be obtained in general since the nonlinear system model cannot be integrated over the sampling time [1] [3]. As an alternative, an approximate discrete time equivalent model of a continuous time system is developed using numerical integration techniques. The most suitable in this regard is the Euler method [1] [3] [4] [5]. In the past it has been shown by the Nesic and Teel in [4] that the control design based on an approximate discrete time model yields larger region of attraction for same sampling time, when compared with the performance of sampled-data control with control design based on a continuous time system model. Sampled-data control of the nonlinear systems has been extensively discussed in [1] [3] and sampled-data linearizing feedback control of a class of a nonlinear systems based on an Euler discretized models has been proposed in [5] 
Quadrotor unmanned Aerial Vehicles (UAVs) have attracted considerable attention of control engineers. This is because of their wide ranging civil as well as military applications such as surveillance, rescue and photography etc. [6] [7] [8] [9]. Moreover, in addition to their ability to take off and land vertically, quadrotors have enhanced payload capability due to their four thrusts generating propellers and are also much simpler to build as compared to an orthodox helicopter [6].

Control of a quadrotor UAV in the continuous time domain has been developing for years. Chattering free altitude control of a quadrotor using sliding mode control (SMC) has been presented in [10]. Samir Boubdallah has presented its attitude stabilization using a continuous time SMC which actually suffers from chattering [6] [7]. In [9] and [11] tracking control based on a SMC has been developed for different dynamic models. Continuous time backstepping control of a quadrotor has been suggested in [11] as well. Quadrotor model has been divided into a fully-actuated and an under-actuated subsystem in [12] and a rate bounded PID control along with the SMC has been proposed to stabilize a fully-actuated subsystem. A comparative analysis between an adaptive SMC and a feedback linearization in the presence of modelling uncertainties has been carried out in [13] and it has been shown that the SMC is more robust than the feedback linearizing control. In [14] an extended observer has been used to estimate a class of disturbances and a continuous time SMC has been applied to stabilize the attitude of a quadrotor in the presence of these disturbances. An integral SMC has been used in [11] for the attitude stabilization of a quadrotor UAV. [6-8], [15] and [16] proposed the backstepping control of a quadrotor. Where [15] discussed the command filtered backstepping control of a quadrotor to track the attitude trajectory and [16] has achieved the stabilizing backstepping control of a quadrotor UAV by dividing the dynamic model in three subsystems such as underactuated, fully-actuated and propeller subsystems. In [17] $L_{1}-$ Optimal control of a quadrotor has been discussed. Feedback linearizing control of a quadrotor UAV along with a sliding mode observer has been presented in [18] which reject the parametric uncertainties.

The literature for the discrete time control of a quadrotor UAV is yet to be developed. This paper presents the sampled-data control of a quadrotor UAV using backstepping technique and a comparison between two sampled-data approaches. It has been shown by the simulations that the proposed discrete time control based on an Euler approximate model is far more robust to the wind gust and modelling uncertainties as compared to the sampled-data control based on a continuous time system model.

This paper is organized as follows; brief description of the dynamic model of a quadrotor UAV is presented in section-2. Section-3 presents the sampled-data backstepping control based on a continuous time system model. Whereas, Sampled-data backstepping control based on an Euler approximate model has been presented in section-4. Simulations in the presence of modelling uncertainties and wind gust have been carried out to present a comparative analysis between the two sampled-data approaches in section- 5 followed by the conclusion in section- 6 .

\section{SYSTEM MODEL}

Quadrotor is an under-actuated electromechanical system with six degrees of freedom and four control inputs. These inputs are the thrusts generated by the counter rotating pairs of propellers attached at the corners of the X-shaped frame [6] [7] [8] [9] [10] [11]. The thrust generated can be used to produce three angular motions, roll $(\varnothing)$, pitch $(\theta)$ and yaw $(\varphi)$ and three translational motions along $x, y$ and $z$-axis respectively as described in [6] [7] [8] [9] [10] [11]. These angular motions are measured in the body fixed frame. On the other hand, the translational motion of a quadrotor is modelled by transforming the forces acting on a quadrotor to the earth fixed frame by the means of a transformation matrix " $R$ " [6] [7] [8] [9] [10] [11].

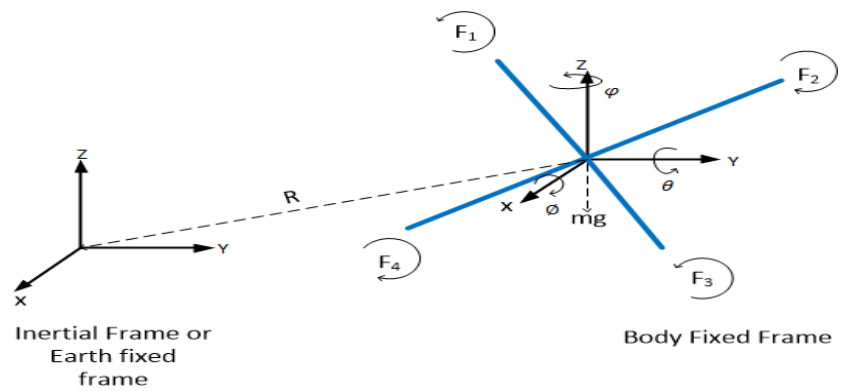

Figure1. Quadrotor free body diagram 
The dynamic model of quadrotor as presented in [6], [7] and [8] is,

$$
\begin{aligned}
& \ddot{\emptyset}=\dot{\theta} \dot{\varphi}\left(\frac{I_{y}-I_{z}}{I_{x}}\right)-\frac{J_{r}}{I_{x}} \dot{\theta} \Omega+\frac{l}{I_{x}} U_{2} \\
& \ddot{\theta}=\dot{\varnothing} \dot{\varphi}\left(\frac{I_{z}-I_{x}}{I_{y}}\right)-\frac{J_{r}}{I_{y}} \dot{\theta} \Omega+\frac{l}{I_{y}} U_{3} \\
& \ddot{\varphi}=\dot{\emptyset} \dot{\theta}\left(\frac{I_{x}-I_{y}}{I_{z}}\right)+\frac{l}{I_{z}} U_{4} \\
& \ddot{x}=(\cos \emptyset \sin \theta \cos \varphi-\sin \emptyset \sin \varphi) \frac{1}{m} U_{1} \\
& \ddot{y}=(\cos \emptyset \sin \theta \sin \varphi-\sin \emptyset \cos \varphi) \frac{1}{m} U_{1} \\
& \ddot{z}=-g+(\cos \emptyset \cos \theta) \frac{1}{m} U_{1}
\end{aligned}
$$

Where, $I_{x}, I_{y}$ and $I_{z}$ are the moments of inertia along $\mathrm{x}, \mathrm{y}$ and $\mathrm{z}$ axis respectively. $J_{R}, l$ and $m$ represent rotor inertia, length of the rod and mass of vehicle respectively. And, $\varnothing, \theta$ and $\varphi$ are respectively roll, pitch and yaw angles in body fixed frame and $x, y$ and $z$ are linear positions with respect to an inertial frame of reference. The thrust and drag factors are $b$ and $d$ respectively.

The control inputs are,

$$
\begin{aligned}
& U_{1}=b\left({\Omega_{1}}^{2}+{\Omega_{2}}^{2}+{\Omega_{3}}^{2}+{\Omega_{4}}^{2}\right) \\
& U_{2}=b\left(\Omega_{4}{ }^{2}-\Omega_{2}{ }^{2}\right) \\
& U_{3}=b\left(\Omega_{3}{ }^{2}-{\Omega_{1}}^{2}\right) \\
& U_{4}=d\left({\Omega_{2}}^{2}+{\Omega_{4}}^{2}-{\Omega_{3}}^{2}-{\Omega_{1}}^{2}\right)
\end{aligned}
$$

Where, $\Omega_{i}$ is the speed of rotation of each propeller and

$$
\Omega=\Omega_{2}+\Omega_{4}-\Omega_{1}-\Omega_{3}
$$

Representing (1) in state space we have a dynamic model of a quadrotor as,

$$
\begin{aligned}
\dot{x}_{1} & =x_{2} \\
\dot{x}_{2} & =x_{4} x_{6} a_{1}+x_{4} a_{2} \Omega+b_{1} U_{2} \\
\dot{x}_{3} & =x_{4} \\
\dot{x}_{4} & =x_{2} x_{6} a_{3}+x_{2} a_{4} \Omega+b_{2} U_{3} \\
\dot{x}_{5} & =x_{6} \\
\dot{x}_{6} & =x_{2} x_{4} a_{5}+b_{3} U_{4} \\
\dot{x}_{7} & =x_{8} \\
\dot{x}_{8} & =-g+\left(\cos x_{1} \cos x_{3}\right) \frac{U_{1}}{m} \\
\dot{x}_{9} & =x_{10} \\
\dot{x}_{10} & =\frac{1}{m} U_{x} U_{1} \\
\dot{x}_{11} & =x_{12} \\
\dot{x}_{12} & =\frac{1}{m} U_{y} U_{1}
\end{aligned}
$$

Where $x_{1}, x_{3}, x_{5}$ represent the roll, pitch, yaw and $x_{2}, x_{4}, x_{6}$ are their time derivatives respectively. Altitude, $\mathrm{x}$-position and $\mathrm{y}$-position are represented by $x_{7}, x_{9}, x_{11}$ and their time derivatives are denoted by $x_{8}, x_{10}$, and $x_{12}$ respectively.

And $a_{1}=\left(I_{y}-I_{z}\right) / I_{x}, a_{2}=-J_{R} / I_{x}, a_{3}=\left(I_{z}-I_{x}\right) / I_{y}, a_{4}=J_{R} / I_{y}, a_{5}=\left(I_{x}-I_{y}\right) / I_{z}$ $b_{1}=l / I_{x}, b_{2}=l / I_{y}, b_{3}=l / I_{z}$

$$
U_{x}=\cos x_{1} \sin x_{3} \cos x_{5}+\sin x_{1} \sin x_{5}
$$

$U_{y}=\cos x_{1} \sin x_{3} \sin x_{5}-\sin x_{1} \cos x_{5}$ 


\section{SAMPLED-DATA CONTROL BASED ON A CONTINUOUS TIME SYSTEM MODEL}

With this approach a controller is extracted by the discretization of a continuous time control obtained from a continuous time system model. Continuous time backstepping control of a quadrotor UAV has been developed in [6] [7] [8] [11] [12] [13]. We proceed with the control developed by Samir Boubdallah in [6] and [7].

$$
U_{2}=\frac{1}{b_{1}}\left[e_{1}-x_{4} x_{6} a_{1}-x_{4} a_{2} \Omega-\alpha_{1}\left(e_{2}+\alpha_{1} e_{1}\right)-\alpha_{2} e_{2}\right] \quad ; \alpha_{i}>0
$$

Where $e_{1}$ and $e_{2}$ as assumed in [6] and [7] are

$$
\begin{aligned}
& e_{1}=x_{1 d}-x_{1} \\
& e_{2}=x_{2}-x_{1 d}-\alpha_{1} e_{1}
\end{aligned}
$$

Similarly for remaining control inputs we have,

$$
\begin{aligned}
& U_{3}=\frac{1}{b_{2}}\left[e_{3}-x_{2} x_{6} a_{3}-x_{2} a_{4} \Omega-\alpha_{3}\left(e_{4}+\alpha_{3} e_{3}\right)-\alpha_{4} e_{4}\right] \\
& U_{4}=\frac{1}{b_{3}}\left[e_{5}-x_{2} x_{4} a_{5}-\alpha_{5}\left(e_{6}+\alpha_{5} e_{5}\right)-\alpha_{6} e_{6}\right] \\
& U_{1}=\frac{m}{\cos x_{1} \cos x_{3}}\left[e_{7}+g-\alpha_{7}\left(e_{8}+\alpha_{7} e_{7}\right)-\alpha_{8} e_{8}\right] \\
& U_{x}=\frac{m}{U_{1}}\left[e_{9}-\alpha_{9}\left(e_{10}+\alpha_{9} e_{9}\right)-\alpha_{10} e_{10}\right] \\
& U_{y}=\frac{m}{U_{1}}\left[e_{11}-\alpha_{11}\left(e_{12}+\alpha_{11} e_{11}\right)-\alpha_{12} e_{12}\right]
\end{aligned}
$$

Where, $e_{3}=x_{3 d}-x_{3}, e_{5}=x_{5 d}-x_{5}, e_{7}=x_{7 d}-x_{7}, e_{9}=x_{9 d}-x_{9}, e_{11}=x_{11 d}-x_{11}$ and $\alpha_{i}>0$

Since $x$ and $y$ motions of a quadrotor can be achieved by rotating the vehicle at a desired pitch and roll angles respectively. Therefore, using (4), (5), (10) and (11) we can extract the desired reference angles for $x$ and $y$-positions as,

$$
\emptyset=\sin ^{-1}\left(U_{x} \sin \varphi-U_{y} \cos \varphi\right)
$$

$$
\theta=\sin ^{-1}\left(\frac{U_{x} \sin \varphi+U_{y} \cos \varphi}{\cos \varnothing}\right)
$$

Discretization of a continuous time control with the sampling time " $T$ " gives us,

$$
\begin{aligned}
& U_{2}[k]=\frac{1}{b_{1}}\left[e_{1}[k]-x_{4}[k] x_{6}[k] a_{1}-x_{4}[k] a_{2} \Omega-\alpha_{1}\left(e_{2}[k]+\alpha_{1} e_{1}[k]\right)-\alpha_{2} e_{2}[k]\right] \\
& U_{3}[k]=\frac{1}{b_{2}}\left[e_{3}[k]-x_{2}[k] x_{6}[k] a_{3}-x_{2}[k] a_{4} \Omega-\alpha_{3}\left(e_{4}[k]+\alpha_{3} e_{3}[k]\right)-\alpha_{4} e_{4}[k]\right] \\
& U_{4}[k]=\frac{1}{b_{3}}\left[e_{5}[k]-x_{2}[k] x_{4}[k] a_{5}-\alpha_{5}\left(e_{6}[k]+\alpha_{5} e_{5}[k]\right)-\alpha_{6} e_{6}[k]\right] \\
& U_{1}[k]=\frac{m}{\cos x_{1}[k] \cos x_{3}[k]}\left[e_{7}[k]+g-\alpha_{7}\left(e_{8}[k]+\alpha_{7} e_{7}[k]\right)-\alpha_{8} e_{8}[k]\right] \\
& U_{x}[k]=\frac{m}{U_{1}[k]}\left[e_{9}[k]-\alpha_{9}\left(e_{10}[k]+\alpha_{9} e_{9}[k]\right)-\alpha_{10} e_{10}[k]\right] \\
& U_{y}[k]=\frac{m}{U_{1}[k]}\left[e_{11}[k]-\alpha_{11}\left(e_{12}[k]+\alpha_{11} e_{11}[k]\right)-\alpha_{12} e_{12}[k]\right]
\end{aligned}
$$

\section{SAMPLED-DATA CONTROL BASED ON AN EULER APPROXIMATE MODEL}

Sampled-data control based on a continuous time system model can yield satisfactory performance only for a sufficiently small sampling time, but in practical scenario too small a sampling time cannot be 
chosen due to the limitations imposed by the processing speed of computers [1] [3]. Therefore a more reliable strategy is adopted that not only takes account of sampling time from the very first step of the control design, but also yields an improved region of attraction in states [1] [3] [4]. An approximate discrete time representation of a quadrotor model has been obtained using an Euler forward difference method as suggested in [1] [3] [4] [5] which actually keeps the structure of a nonlinear system intact.

$$
\begin{aligned}
& x_{1 a}[k+1]=x_{1 a}[k]+T\left(x_{2 a}[k]\right) \\
& x_{2 a}[k+1]=x_{2 a}[k]+T\left(x_{4 a}[k] x_{6 a}[k] a_{1}+x_{4 a}[k] a_{2} \Omega+b_{1} U_{2}[k]\right) \\
& x_{3 a}[k+1]=x_{3 a}[k]+T\left(x_{4 a}[k]\right) \\
& x_{4 a}[k+1]=x_{4 a}[k]+T\left(x_{2 a}[k] x_{6 a}[k] a_{3}+x_{2 a}[k] a_{4} \Omega+b_{2} U_{3}[k]\right) \\
& x_{5 a}[k+1]=x_{5 a}[k]+T\left(x_{6 a}[k]\right) \\
& x_{6 a}[k+1]=x_{6 a}[k]+T\left(x_{2 a}[k] x_{4 a}[k] a_{5}+b_{3} U_{4}[k]\right) \\
& x_{7 a}[k+1]=x_{7 a}[k]+T\left(x_{8 a}[k]\right) \\
& x_{8 a}[k+1]=x_{8 a}[k]+T\left(-g+\left(\cos x_{1 a}[k] \cos x_{3 a}[k]\right) \frac{U_{1}}{m}\right) \\
& x_{9 a}[k+1]=x_{9 a}[k]+T\left(x_{10 a}[k]\right) \\
& x_{10 a}[k+1]=x_{10 a}[k]+T\left(\left(\cos x_{1 a}[k] \sin x_{3 a}[k] \cos x_{5 a}[k]+\sin x_{1 a}[k] \sin x_{5 a}[k]\right) \frac{U_{1}}{m}\right) \\
& x_{11 a}[k+1]=x_{11 a}[k]+T\left(x_{12 a}[k]\right) \\
& x_{12 a}[k+1]=x_{12 a}[k]+T\left(\left(\cos x_{1 a}[k] \sin x_{3 a}[k] \sin x_{5 a}[k]+\sin x_{1 a}[k] \cos x_{5 a}[k]\right) \frac{U_{1}}{m}\right)
\end{aligned}
$$

Subscript "a" represents an approximate model. In order to stabilize the roll angle we define the error dynamics as

$$
e_{1}(k)=x_{1 d}(k)-x_{1 a}(k)
$$

And apply the discrete time Lyapunov analysis

$$
\begin{aligned}
& V_{1}\left(e_{1}[k]\right)=e_{1}{ }^{2}[k] \\
& \begin{aligned}
\Delta V_{1}\left(e_{1}[k]\right) & =V_{1}[k+1]-V_{1}[k] \\
& =\left(e_{1}[k+1]+e_{1}[k]\right)\left(\Delta x_{1 d}[k]-T x_{2 a}[k]\right)
\end{aligned}
\end{aligned}
$$

$T x_{2 a}$ Can be viewed as the input to stabilize $e_{1}[k]$

$$
T x_{2 a}=\Delta x_{1 d}[k]+\alpha_{1}\left(e_{1}[k+1]+e_{1}[k]\right)
$$

Where $\left(e_{1}[k+1]+e_{1}[k]\right)$ is the sum of current and previous values of error, to backstep we use the change of variables [19],

$$
e_{2}[k]=T x_{2 a}[k]-\Delta x_{1 d}[k]-\alpha_{1}\left(e_{1}[k+1]+e_{1}[k]\right)
$$

Taking composite Lyapunov function as

$$
\begin{aligned}
V_{2}\left(e_{1}[k], e_{2}[k]\right) & =e_{1}{ }^{2}[k]+e_{2}{ }^{2}[k] \\
\Delta V_{2}\left(e_{1}[k], e_{2}[k]\right) & =\left(e_{1}{ }^{2}[k+1]-e_{1}{ }^{2}[k]\right)+\left(e_{2}{ }^{2}[k+1]-e_{2}{ }^{2}[k]\right)
\end{aligned}
$$

Expanding (26) helps us to reach

$$
\begin{aligned}
& \Delta V_{2}= \\
& -e_{2}[k]\left(e_{1}[k+1]+e_{1}[k]\right)-\alpha_{1}\left(e_{1}[k+1]+e_{1}[k]\right)^{2}+ \\
& \left(e_{2}[k+1]+e_{2}[k]\right)\left[T^{2}\left(x_{4 a}[k] x_{6 a}[k] a_{1}+x_{4 a}[k] a_{2} \Omega+b_{1} U_{2}[k]\right)-\Delta^{2} x_{1 d}[k]+\alpha_{1}\left(e_{1}[k]-\right.\right. \\
& \left.\left.e_{1}[k+2]\right)\right]
\end{aligned}
$$

Thus, the control input $U_{2}[k]$ that ensures the negative definiteness/semi definiteness of Lyapunov difference can be taken as, 


$$
\begin{aligned}
& U_{2}=\frac{1}{b_{1}}\left[-x_{4 a}[k] x_{6 a}[k] a_{1}-x_{4 a}[k] a_{2} \Omega-\alpha_{2}\left(e_{2}[k+1]+e_{2}[k]\right)-T c_{1} \alpha_{1}\left(e_{1}[k]-e_{1}[k+2]\right)+\right. \\
& \left.T c_{1} \alpha_{2} e_{2}[k]\left(\frac{e_{1}[k+1]+e_{1}[k]}{e_{2}[k+1]+e_{2}[k]}\right)\right]
\end{aligned}
$$

Working on the same lines for the other states we reach to the following set of control inputs,

$$
\begin{aligned}
& U_{3}=\frac{1}{b_{2}}\left[-x_{2 a}[k] x_{6 a}[k] a_{3}-x_{2 a}[k] a_{4} \Omega-\alpha_{4}\left(e_{4}[k+1]+e_{4}[k]\right)-T c_{2} \alpha_{3}\left(e_{3}[k]-e_{3}[k+2]\right)+\right. \\
& \left.T c_{2} \alpha_{4} e_{4}[k]\left(\frac{e_{3}[k+1]+e_{3}[k]}{e_{4}[k+1]+e_{4}[k]}\right)\right] \\
& U_{4}=\frac{1}{b_{3}}\left[-x_{2 a}[k] x_{4 a}[k] a_{5}-\alpha_{6}\left(e_{6}[k+1]+e_{6}[k]\right)-T c_{3} \alpha_{5}\left(e_{5}[k]-e_{5}[k+2]\right)+\right. \\
& \left.T c_{3} \alpha_{6} e_{6}[k]\left(\frac{e_{5}[k+1]+e_{5}[k]}{e_{6}[k+1]+e_{6}[k]}\right)\right] \\
& U_{1}= \\
& \frac{m}{\cos x_{1 a}[k] \cos x_{3 a}[k]}\left[g-\alpha_{8}\left(e_{8}[k+1]+e_{8}[k]\right)-T c_{4} \alpha_{7}\left(e_{7}[k]-e_{7}[k+2]\right)+\right. \\
& \left.T c_{4} \alpha_{8} e_{8}[k]\left(\frac{e_{7}[k+1]+e_{7}[k]}{e_{8}[k+1]+e_{8}[k]}\right)\right] \\
& U_{x}= \\
& \frac{m}{U_{1}}\left[-\alpha_{10}\left(e_{10}[k+1]+e_{10}[k]\right)-T c_{5} \alpha_{9}\left(e_{9}[k]-e_{9}[k+2]\right)+T c_{5} \alpha_{10} e_{10}[k]\left(\frac{e_{9}[k+1]+e_{9}[k]}{e_{10}[k+1]+e_{10}[k]}\right)\right] \\
& \begin{array}{l}
U_{y}= \\
\frac{m}{U_{1}}\left[-\alpha_{12}\left(e_{12}[k+1]+e_{12}[k]\right)-T c_{6} \alpha_{11}\left(e_{11}[k]-e_{11}[k+2]\right)+T c_{6} \alpha_{12} e_{12}[k]\left(\frac{e_{11}[k+1]+e_{11}[k]}{e_{12}[k+1]+e_{12}[k]}\right)\right]
\end{array}
\end{aligned}
$$

$\alpha_{i}>0$, and $c_{i}$ is a large positive constant.

\section{RESULTS AND ANALYSIS}

Simulations have been carried out for the sampling time of $10 \mathrm{~ms}$ with model parameters taken from [6]. In these simulations quadrotor is required to reach the final destination of $\{2,2,2\}$ while stabilizing the yaw angle $(\varphi)$ to zero. The initial condition for the rotational subsystem is $[\varnothing, \theta, \varphi]=\left[0,0, \frac{\pi}{16}\right]$.

\subsection{Simulations with Different Initial Conditions}

Both the designs are simulated for the initial condition of $[\varnothing, \theta, \varphi]=\left[0,0, \frac{\pi}{16}\right]$ and a full state stabilization is achieved. Sampled-data control based on a continuous time model destabilizes the system for a specific set of gains at $[\varnothing, \theta, \varphi]=\left[0,0, \frac{\pi}{4}\right]$ whereas, the design based on a discrete time equivalent model still stabilizes the quadrotor UAV. This shows that the proposed sampled-data backstepping control based on an approximate discrete time model has a larger region of attraction as compared to the sampled-data backstepping control based on a continuous time system model.

\subsection{Simulations with Perturbed Parameters}

Robustness of the controller is the measure of insensitivity to the parameter variations. For that purpose Monte Carlo method for the simulations is adopted here. In these simulations a quadrotor's parameter including mass, moment of inertia along three the axis, rotor inertia, thrust and drag factor are perturbed in random with some tolerance and a simulation is run for each randomly perturbed set of parameters. This process is repeated for one thousand times. Quadrotor length is kept constant in these simulations. Simulations have revealed that the controller obtained by discretizing a continuous time control fails to stabilize the system for each set of a randomly perturbed parameter even with the tolerance of \pm 3 percent. Whereas our sampled-data control based on an Euler approximate model stabilizes the system even 
with the random perturbations of \pm 30 percent tolerance. Upper and lower bound for each state have been presented to help you visualize the robustness of the proposed control.

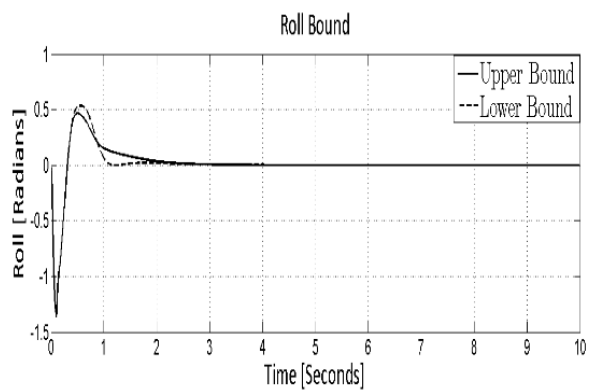

(a)

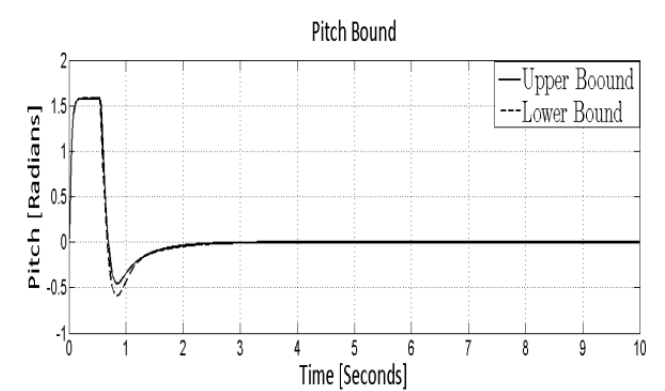

(b)

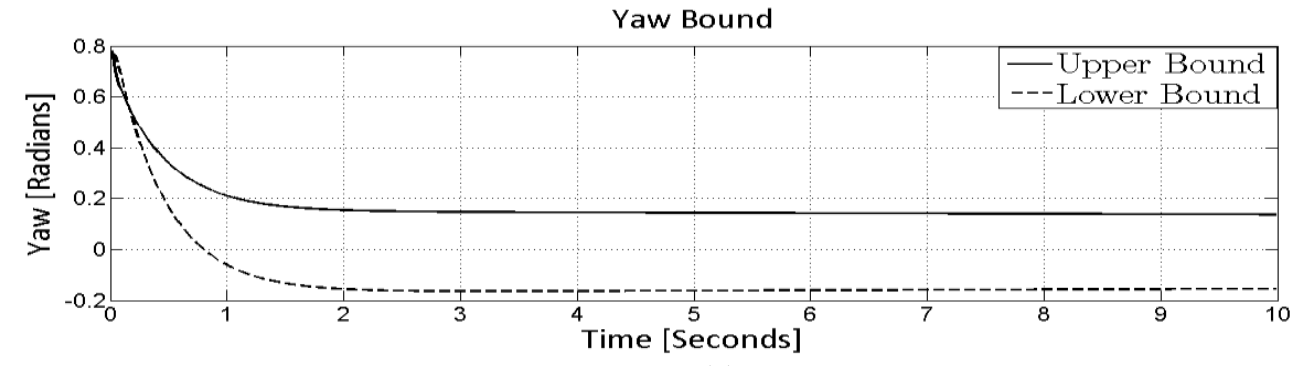

(c)

Figure 2. Sampled-Data Backstepping Control Based on An Approximate Discrete Time System Model. With Random Perturbation of \pm 30 Percent Tolerance (a) Roll Bound (b) Pitch Bound (c) Yaw Bound.

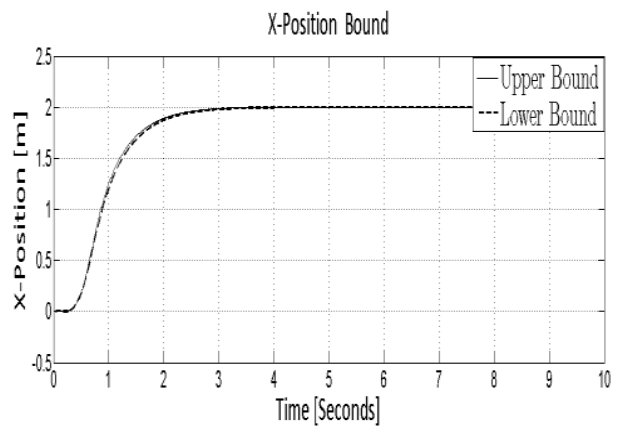

(a)

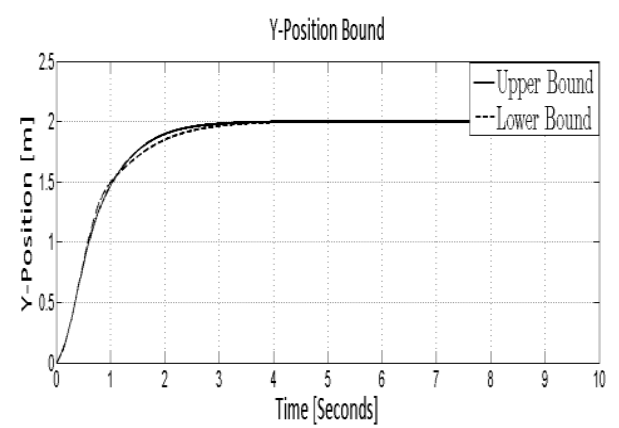

(b)

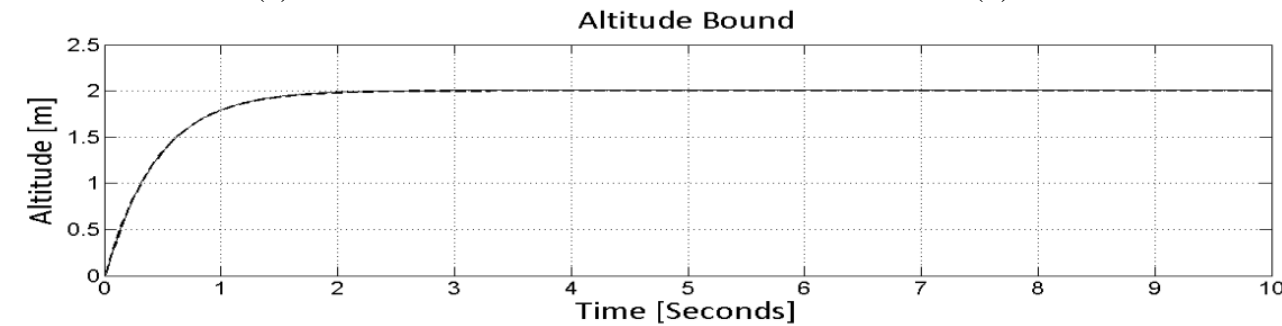

(c)

Figure 3. Sampled-Data Backstepping Control Based on An Approximate Discrete Time System Model. With Random Perturbation of \pm 30 Percent Tolerance (a) X-Position Bound (b) Y-Position Bound (c) Altitude Bound.

\subsection{Simulations with Wind Gusts and Nominal Parameters}

One of the environmental effects upon any aerial vehicle is the wind gust that some time may destabilize the system. Therefore, an effort is made to testify the robustness of both the discrete time 
controllers in the presence of the wind gust. The wind gust profile is generated by the Simulink's "Aerospace Blockset" library. Start time for the wind gust is zero seconds while it gradually establishes in 10 meters length at the maximum speed of 9 meters per second. Both the controllers encounter the same profile of wind gust along the $\mathrm{x}$ and $\mathrm{y}$-axis simultaneously and it is quite clear from the simulations that discrete time control based on an Euler approximate model can smoothly withstand the applied wind gust profile and is able to stabilize with negligibly small steady state error. While the discrete time controller based on a continuous time system model destabilizes in the presence of the wind gust of the same profile since the drift along $\mathrm{x}$ and $y$-axis is in thousands of meters. The vehicle has to reach the position of $[2,2,2]$ meters while stabilizing the yaw to zero.

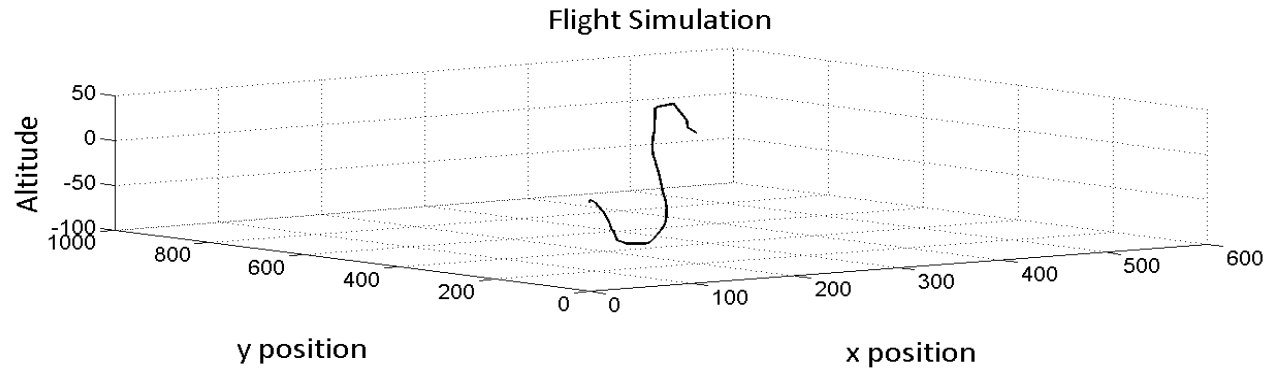

(a)

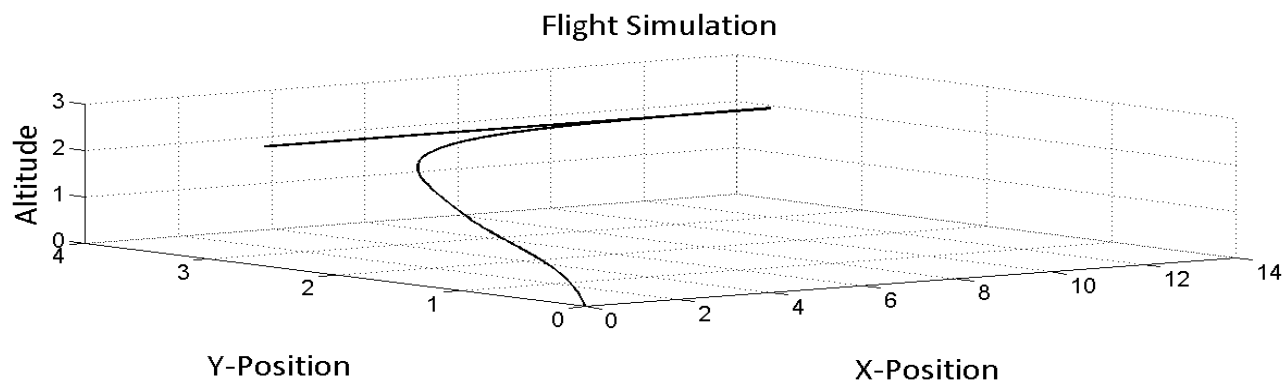

(b)

Figure 4. Flight Simulations In The Presence of Wind Gust. (a) Sampled-Data Backstepping Control Based on a Continuous Time System Model (b) Sampled-Data Backstepping Control Based on Approximate Discrete Time System Model.

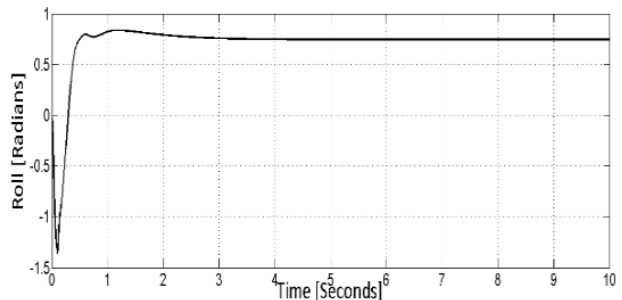

(a)

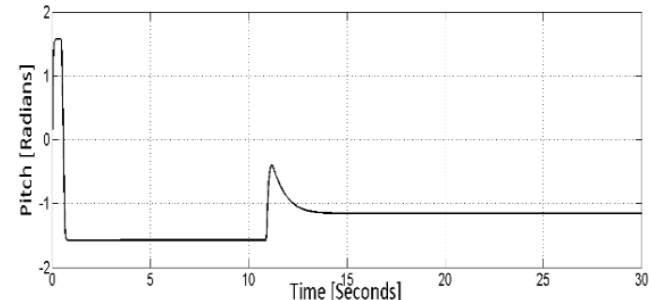

(b)

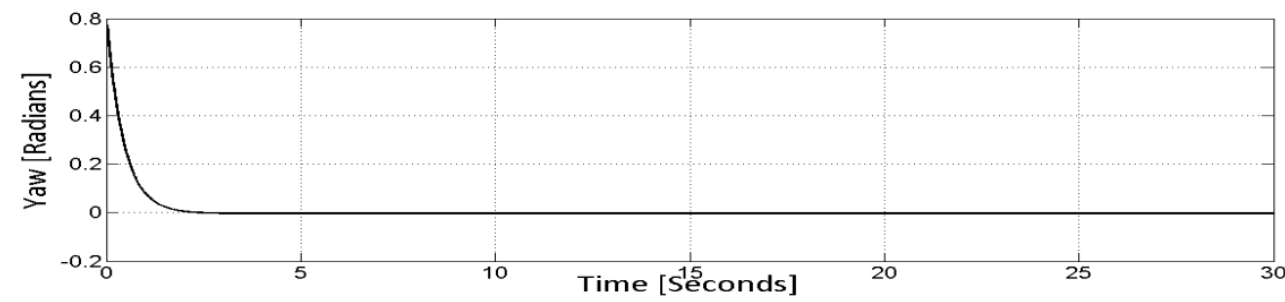

(c)

Figure 5. Sampled-Data Backstepping Control Based on An Approximate Discrete Time System Model. With Wind Gust (a) Roll (b) Pitch (c) Yaw. 


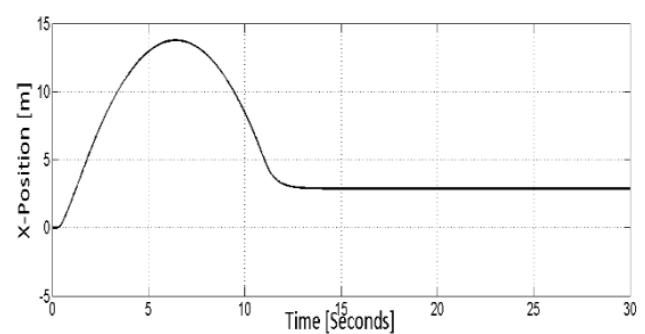

(a)

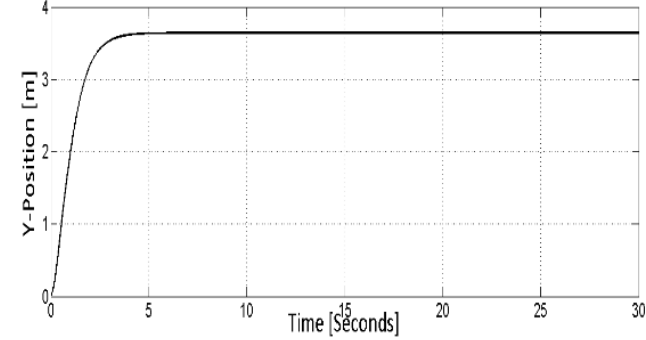

(b)

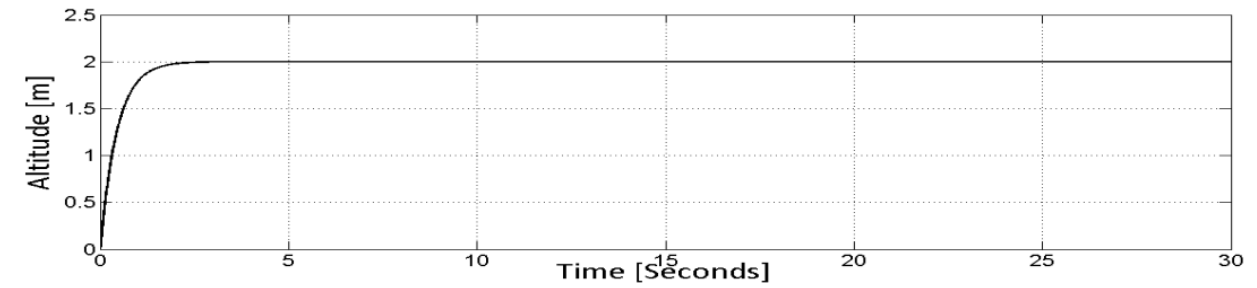

(c)

Figure 6. Sampled-Data Backstepping Control Based on An Approximate Discrete Time System Model. With Wind Gust (a) X-Position (b) Y-Position (c) Altitude.

\subsection{Simulation Results with Perturbed Parameters and Wind Gusts}

Finally, both the controllers are subject to the parameter perturbation in the presence of the wind gust already described above. Sampled-data backstepping based on a continuous time system model again destabilizes the system in the presence of wind gust even with the random perturbation of \pm 3 percent tolerance. On the other hand, the proposed discrete time control based on a discrete time equivalent system model successfully stabilizes the system in the presence of wind gust up to the random perturbation of \pm 15 percent. Simulations are again performed for one thousand times. The maximum and the minimum bounds are plotted only.
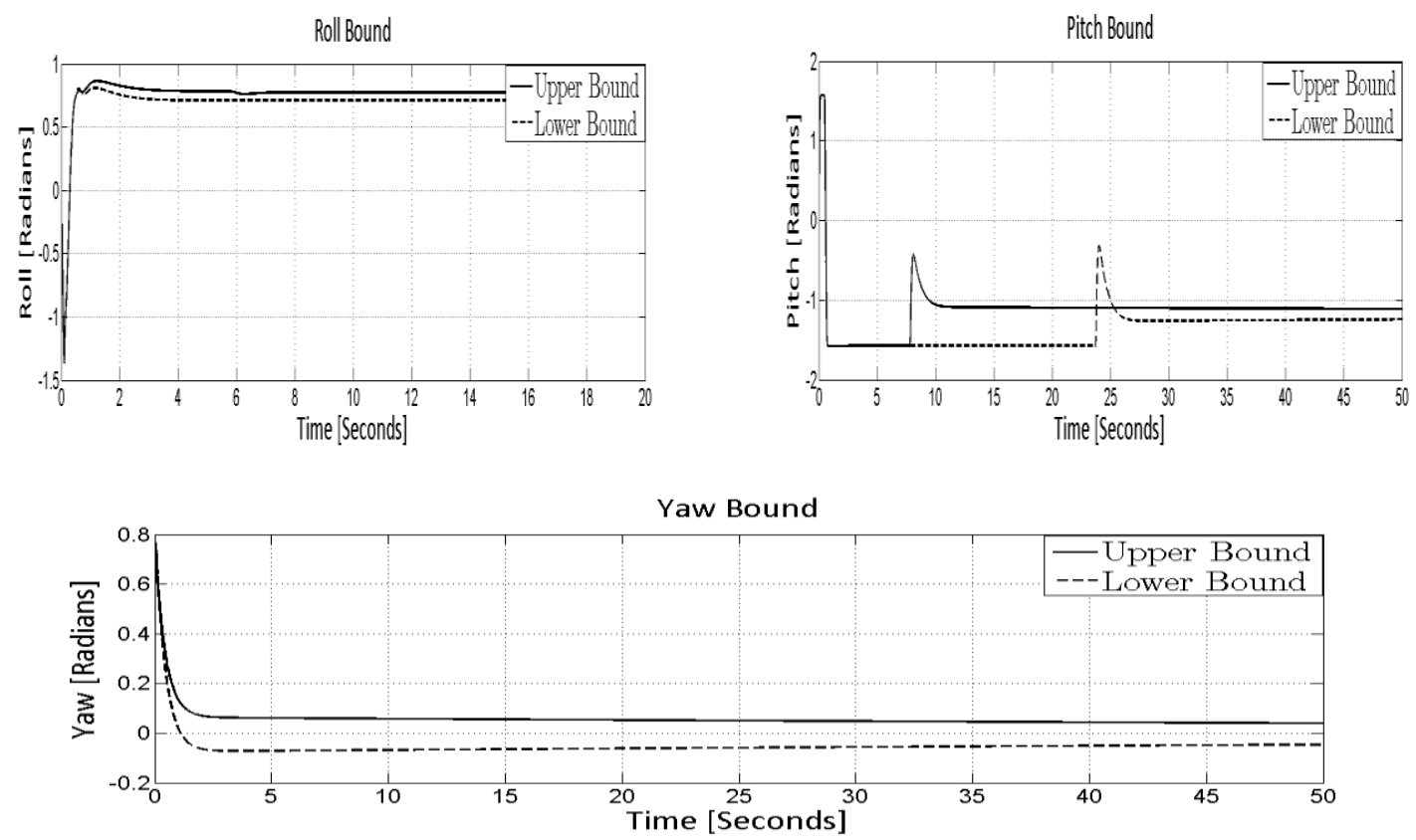

(c)

Figure 7. Sampled-Data Backstepping Control Based on Approximate Discrete Time Equivalent System Model. With Random Perturbation of \pm 15 Percent Tolerance and Wind Gust (a) Roll Bound (b) Pitch

Bound(c) Yaw Bound. 


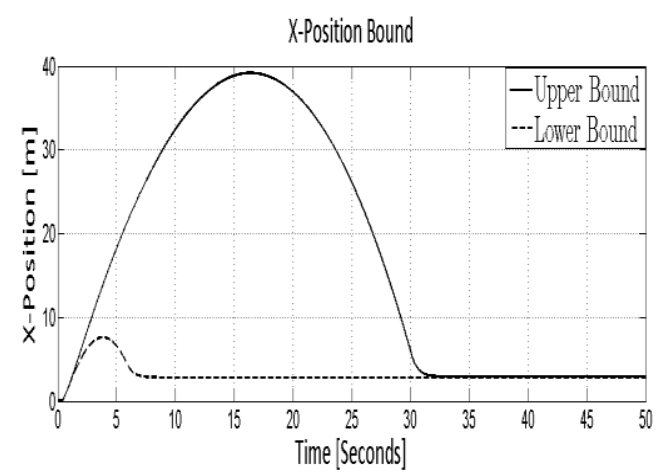

(a)

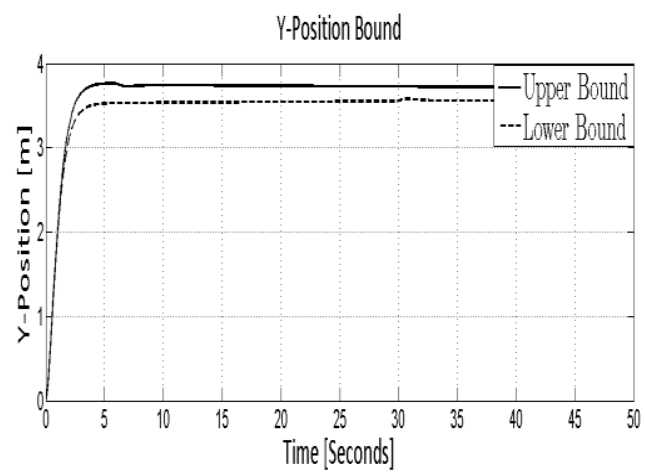

(b)

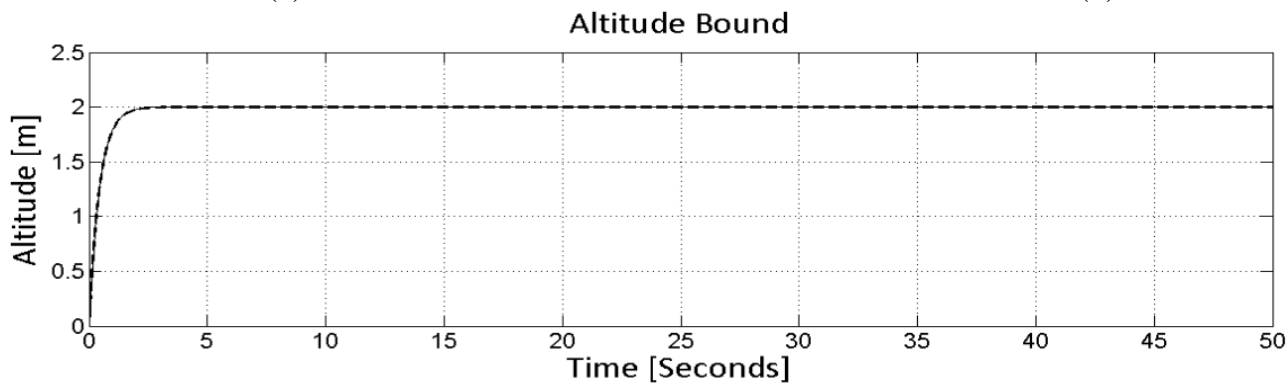

(c)

Figure8. Sampled-Data Backstepping Control Based on An Approximate Discrete Time System Model. With Random Perturbation of \pm 15 Percent Tolerance and Wind Gust (a) X-Position Bound (b) Y-Position Bound (c) Altitude Bound.

\section{CONCLUSION}

A novel backstepping control of a quadrotor UAV has been presented that completely stabilizes the aircraft in a semiglobal practical sense. Simulations for different initial conditions have shown that controller designed on the basis of an approximate discrete time system model has a larger region of attraction as compared to controller obtained by discretization of a continuous time control. Furthermore, Monte Carlo simulations have revealed that sampled-data backstepping control based on an Euler approximate model can stabilize the quadrotor UAV with random parameter perturbations of \pm 30 percent tolerance. Whereas, sampled-data backstepping control based on a continuous time system model destabilize the system even with random parameter perturbations of \pm 3 percent tolerance. Additionally, the proposed sampled-data backstepping control based on an Euler approximate model stabilizes the quadrotor UAV in the presence of wind gust and random parameter perturbations of \pm 15 percent tolerance. On the other hand, sampled-data backstepping control based on a continuous time system model again destabilizes the system with simultaneous presence of wind gust and random parameter perturbations of \pm 3 percent tolerance. Thus, the Proposed discrete time backstepping control based on an Euler approximate model is far more robust to the modelling uncertainties and exogenous noise such as wind gust of some moderate speed as compared to the controller obtained by discretizing a continuous time backstepping control.

\section{REFERENCES}

[1] D. S. Laila., "Design and Analysis of Nonlinear Sampled Data Control Systems", PhD Dissertation-Department of Electrical and Electronics Engineering, the University of Melbourne, April 2003.

[2] K. Ogata., "Discrete-Time Control Systems", $2^{\text {nd }}$ Edition (Prentice-Hall International Inc.)

[3] F. M. Malik, "Sampled Data Control Based on Discrete Time Equivalent Models", PhD Dissertation-Department of Electrical Engineering, NUST College of Electrical and Mechanical Engineering, 2009.

[4] D. Nesic, A. R. Teel., "Backstepping on Euler Approximate Model for Stabilization of Sampled Data Nonlinear Systems", Proceedings of the $40^{\text {th }}$ IEEE Conference on Decision and Control, Orlando, Florida USA, December 2001.

[5] F. M. Malik., et al., "Sampled-Data State Feedback stabilization of a Class of Nonlinear Systems Based on Euler Approximation", Asian Journal of Control. Vol. 13, pp. 1719-1728, 2011.

[6] S. Bouabdallah., "Design and Control of Quadrotors with Application to Autonomous Flying", PhD DissertationSwiss federal Institute of Technology Lausanne (EPFL), 2007. 
[7] S. Bouabdallah., and R. Siegwart., "Backstepping and Sliding Mode Techniques Applied to an indoor Micro Quadrotor", Proceedings of the IEEE international Conference on Robotics and Automation, Barcelona, Spain, April 2005.

[8] S. Bouabdallah., and R. Siegwart., "Full Control of Quadrotor", Proceedings of the IEEE/RJS international Conference on intelligent Robots and Systems, San Diego, CA, USA, Oct 29 -Nov 2007.

[9] H.Bouadi., M.Bouchoucha and M. Tadjini., "Sliding Mode Control Based on Backstepping Approach for an UAV Type Quadrotor,. World Academy of Science, Engineering and Technology, 2007.

[10] I. Gonzalez., S. Salazar., and R. Lozano., "Chattering free Sliding Mode Altitude Control for a Quadrotor Aircraft: Real time Application", J Intell Robot Syst (2014), 73:137-155.

[11] P. Adigbli., C. Grand., et al., "Nonlinear Attitude and Position Control of a Micro Quadrotor Using Sliding Mode and Backstepping Techniques", $3^{\text {rd }}$ US-European Competition and workshop on Micro Ariel Vehicle Systems (MAV07) and European Micro Air Vehicle Conference and Flight Competition (EMAV2007), 17-21 September 2007, Toulouse, France.

[12] A. R. Patel., "Modeling and Analysis of Quadrotor Using Sliding Mode Control", 44 ${ }^{\text {th }}$ IEEE Southeastern Symposium on System Theory, University of North Florida, Jacksonville, FL March 11-13, 2012.

[13] D. Lee., et al, "Feedback Linearization vs. Adaptive Sliding Mode Control for a Quadrotor Helicopter", International journal of Control, Automation and systems (2009) 7(3): 419-428

[14] R. Zhang., et al., "Attitude Control of Quadrotor Aircraft Subject to a Class of time-Varying Disturbances”, IET Control Theory and Applications; 2011, Vol. 5, Iss. 9, pp. 1140-1146.

[15] Z. Zuo, "Trajectory tracking control design with command-filtered compensation for a quadrotor", IET Control Theory and Applications; 2010, Vol. 4, Iss. 11, pp. 2343-2355.

[16] T. Madani., A. Benallegue., "Backstepping Control for a Quadrotor Helicopter", Proceedings of the 2006 IEEE/RSJ International Conference on Intelligent Robots and Systems, October 9-15, 2006, Beijing, China.

[17] A. C. Satici., et al., "Robust Optimal Control of Quadrotor UAVs", IEEE Access, Digital Object Identifier 10.1109/Access. 2013.2260749.

[18] A. Mokhtari., A. Benallegue., and Y. Orlov., "Exact Linearization and Sliding Mode Observer for a Quadrotor Unmanned Aerial Vehicle", International Journal of Robotics and Automation, Vol. 21, No. 1, 2006.

[19] H. K. Khalil., "Nonlinear Systems", $3{ }^{\text {rd }}$ Edition (prentice Hall), 2002.

\section{BIOGRAPHIES OF AUTHORS}

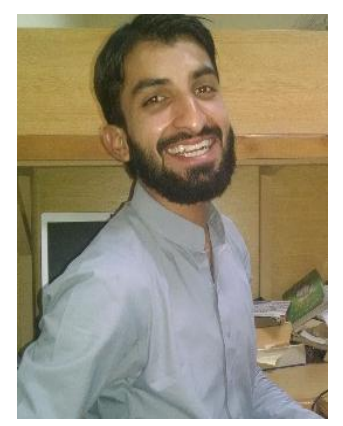

Abdul Jabbar received his B. S degree in Electronics Engineering from COMSATS Institute of Information Technology in 2011 and M.S degree in Electrical Engineering from National University of Sciences and Technology (NUST), College of Electrical and Mechanical Engineering in 2014. His research Interests Include nonlinear control and Robotics.

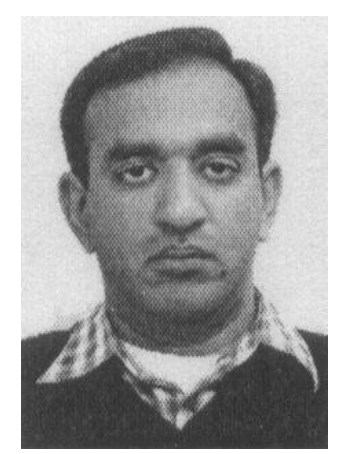

Dr. Fahad Mumtaz Malik received his $\mathrm{PhD}$ in Electrical Engineering from National University of Sciences and Technology (NUST) in 2009. He is currently working as an assistant professor at National University of Sciences and Technology (NUST), College of Electrical and Mechanical Engineering. His Research interests include sampled-data control and flying robots. 